\title{
Pacific
}

Journal of

Mathematics

\section{GENERALIZED NORMAL RULINGS AND INVARIANTS OF LEGENDRIAN SOLID TORUS LINKS}

MikHAIL LAVROV AND DAN RUTHERFORD 


\title{
GENERALIZED NORMAL RULINGS AND INVARIANTS OF LEGENDRIAN SOLID TORUS LINKS
}

\author{
MiKHAIL LAVROV AND DAN RUTHERFORD
}

\begin{abstract}
For Legendrian links in the 1-jet space of $S^{1}$ we show that the 1-graded ruling polynomial may be recovered from the Kauffman skein module. For such links a generalization of the notion of normal ruling is introduced. We show that the existence of such a generalized normal ruling is equivalent to sharpness of the Kauffman polynomial estimate for the ThurstonBennequin number as well as to the existence of an ungraded augmentation of the Chekanov-Eliashberg DGA. Parallel results involving the HOMFLYPT polynomial and 2-graded generalized normal rulings are established.
\end{abstract}

\section{Introduction}

In $\mathbb{R}^{3}$ interesting connections exist between the 2-variable knot polynomials and invariants of Legendrian knots. With respect to the standard contact structure on $\mathbb{R}^{3}$, Fuchs and Tabachnikov [1997] showed that an upper bound for the ThurstonBennequin number arises from the Kauffman and HOMFLY-PT knot polynomials. Furthermore, when this estimate is sharp some nonclassical invariants exhibit nice properties. Specifically, combining results from [Fuchs 2003; Fuchs and Ishkhanov 2004; Sabloff 2005; Rutherford 2006] we have:

Theorem 1.1. For a Legendrian link $L \subset \mathbb{R}^{3}$ the following three statements are all equivalent:

(1) The estimate $\operatorname{tb}(L) \leq-\operatorname{deg}_{a} F_{L}$ (respectively $t b(L) \leq-\operatorname{deg}_{a} P_{L}$ ) is sharp, where $F_{L}, P_{L} \in \mathbb{Z}\left[a^{ \pm 1}, z^{ \pm 1}\right]$ denote the Kauffman and HOMFLY-PT polynomials.

(2) A front diagram for L has a 1-graded (respectively 2-graded) normal ruling.

(3) The Chekanov-Eliashberg DGA of L has a 1-graded (respectively 2-graded) augmentation.

In this article, we establish analogous results for Legendrian knots in the 1-jet space of the circle, $J^{1} S^{1}$. The manifold $J^{1} S^{1}$ is topologically an open solid torus and carries a standard contact structure. Legendrian knots in $J^{1} S^{1}$ have attracted

Lavrov received support from NSF CAREER grant number DMS-0846346.

MSC2010: 57M27, 57R17.

Keywords: Legendrian knot, Kauffman polynomial, skein module, normal ruling. 
a fair amount of attention in the literature; see [Ding and Geiges 2010; Ng and Traynor 2004; Traynor 1997]. The 1-jet space setting comes with convenient projections from which Legendrian knots may be presented via front or Lagrangian diagrams and Legendrian isotopy may be described in a combinatorial manner. 1 -jet spaces also provide a natural setting for the use of generating families.

A convenient formal way to define a normal ruling, $\rho$, of $L$ is as a family of fixed-point-free involutions of the strands of the front diagram of $L$ subject to many restrictions. At least locally, this may be viewed as a decomposition of the front diagram into pairs of paths. Chekanov and Pushkar [2005] introduced normal rulings - albeit with different terminology — as well as related Legendrian isotopy invariants which have become known as ruling polynomials. In connection with augmentations, Fuchs independently defined normal rulings of knots in $\mathbb{R}^{3}$ and, in the case of the Kauffman polynomial, already conjectured the equivalence of (1) and (2) in [Fuchs 2003]. This conjecture was verified in [Rutherford 2006], where it was shown that in fact the 1-graded and 2-graded ruling polynomials appear as coefficients of the Kauffman and HOMFLY-PT polynomials respectively.

Relationships between the Kauffman/HOMFLY-PT invariants and Legendrians knots in $J^{1} S^{1}$ have already begun to be studied, and several factors make the situation more interesting. For instance, the HOMFLY-PT polynomial, $P_{L}$, of a solid torus link, $L$, belongs to a polynomial algebra over $R=\mathbb{Z}\left[a^{ \pm 1}, z^{ \pm 1}\right]$ with a countably infinite number of generators $A_{k}, k \in \mathbb{Z} \backslash\{0\}$; the Kauffman polynomial has a similar form. Chmutov and Goryunov [1997] proved Thurston-Bennequin number estimates analogous to those appearing in (1) of Theorem 1.1 using these many variable Kauffman and HOMFLY-PT polynomials. In the case of the HOMFLY-PT polynomial, it was shown in [Rutherford 2011] that the 2-graded ruling polynomial can be recovered from the HOMFLY-PT polynomial, but this requires first specializing via an $R$-module homomorphism $R\left[A_{ \pm 1}, A_{ \pm 2}, \ldots\right] \rightarrow R$. In the present work we develop analogous results involving the 1 -graded ruling polynomial and the Kauffman skein module. (See Theorems 3.4 and 3.6.)

The need to specialize the Kauffman and HOMFLY-PT invariants in order to recover the ruling polynomials has an interesting consequence. There are many solid torus links where the Kauffman or HOMFLY-PT polynomial estimate is sharp, yet the corresponding ruling polynomial vanishes. As a result, for Legendrians in $J^{1} S^{1}$ some adjustment is required to statement (2) of Theorem 1.1. For this purpose, we introduce a quite natural notion of generalized normal ruling where the fixed-pointfree condition is relaxed. Our main result is the following analog of Theorem 1.1:

Theorem 1.2. Let $L \subset J^{1} S^{1}$ be a Legendrian link.

(1) The estimate $t b(L) \leq-\operatorname{deg}_{a} F_{L}$ (respectively $t b(L) \leq-\operatorname{deg}_{a} P_{L}$ ) is sharp if and only if $L$ has a 1-graded (respectively 2-graded) generalized normal ruling. 
(2) Suppose L has been assigned a $\mathbb{Z} / p$-valued Maslov potential. The ChekanovEliashberg DGA of $L$ has a p-graded augmentation if and only if a front diagram for L admits a p-graded generalized normal ruling.

Remark 1.3. (i) Aside from allowing the more general $p$-graded condition in (2), it is natural to organize the three statements into these two equivalences. Even in $\mathbb{R}^{3}$, the authors do not know of any proof of an implication between the statements about the knot polynomial estimates and existence of augmentations which is able to avoid using normal rulings. There are settings, for instance certain contact lens spaces, where Legendrian contact homology [Licata 2011] and HOMFLYPT polynomial estimates [Cornwell 2012a; 2012b] for $t b$ have been established while an appropriate notion of normal ruling has yet to be formulated. For this reason, establishing a more direct link between Bennequin type inequalities and augmentations could prove interesting.

(ii) For Legendrians in $\mathbb{R}^{3}$, there is another interesting condition connected with the equivalent statements in Theorem 1.1. Specifically, the existence of a 0 -graded normal ruling is equivalent to the existence of a linear at infinity generating family for $L$; see [Chekanov and Pushkar 2005; Fuchs and Rutherford 2011]. This statement remains true in $J^{1} S^{1}$. However, it is interesting to ask if links with 0 -graded generalized normal rulings always admit reasonable (say, linear or quadratic at infinity) generating families. To allow for fixed point strands, it seems necessary to consider generating families $F: E \rightarrow \mathbb{R}$ defined on bundles $E \rightarrow S^{1}$ whose fiber has nontrivial homology. As an example, the basic front $A_{m}$ defined in Section 2A may be generated by a function on an $m$-fold cover of $S^{1}$.

Organization. The article is arranged as follows: In Section 2, we provide the necessary background about normal rulings and the Kauffman and HOMFLY-PT invariants and also introduce generalized normal rulings.

Section 3 runs parallel to the results on the HOMFLY-PT skein module and 2-graded rulings from [Rutherford 2011]. We show how to recover the 1-graded ruling polynomial from an appropriate specialization of the Kauffman skein module. A natural basis for the Kauffman skein module is indexed by partitions, and for this basis we provide an explicit formula for the specialization.

In Section 4 we prove part (1) of Theorem 1.2 by combining the results of Section 3 (and of [Rutherford 2011] for the HOMFLY-PT case) with a linear independence argument.

Section 5 deals with part (2) of Theorem 1.2. For the forward implication we base all of our arguments on linear algebraic results from [Barannikov 1994], from which the reason behind the normality conditions, with or without fixed points, becomes clear. 


\section{Background on Legendrian solid torus links}

We assume familiarity with basic concepts about Legendrian knots such as front projections, Legendrian Reidemeister moves, Thurston-Bennequin number, and rotation number, at least for knots in $\mathbb{R}^{3}$. See, for instance, [Geiges 2008], and also note that [Rutherford 2011] contains an alternate discussion of the case of Legendrian knots in $J^{1} S^{1}$.

We view the 1 -jet space of the circle, $J^{1} S^{1}$, as $S^{1} \times \mathbb{R}^{2}$ equipped with the contact structure $\xi=\operatorname{ker}(d z-y d x)$, where $x$ is a circle-valued coordinate. We occasionally refer to a (Legendrian) link $L \subset J^{1} S^{1}$ as a (Legendrian) solid torus link. The front projection of a Legendrian solid torus link consists of some number of closed curves in the $x z$-annulus which we view as $[0,1] \times \mathbb{R}$ with the identification $(0, z) \sim(1, z)$. Generically, front projections are immersed and embedded except at semicubical cusps and transverse double points, and two such projections represent Legendrian isotopic links if and only if they are related by a sequence of Legendrian Reidemeister moves.

We make the convention of extending the Thurston-Bennequin number to homologically nontrivial links by using the front projection formula

$$
t b(L)=w(L)-c(L),
$$

where $w(L)$ denotes the writhe of $L$ (a signed sum of crossings) and $c(L)$ is half the number of cusps of $L$.

Similarly, for a Legendrian knot $L \subset J^{1} S^{1}$ we define the rotation number as

$$
r(L)=\frac{1}{2}(d(L)-u(L)),
$$

where $d(L)$ denotes the number of downward oriented cusps and $u(L)$ the number of upward oriented cusps.

2A. Products of basic fronts. Given two annular front diagrams, $K$ and $L$, we define the product, $K \cdot L$, by stacking $K$ above $L$. In contrast to the case of smooth knot diagrams, this product is noncommutative as the Legendrian isotopy types of $K \cdot L$ and $L \cdot K$ will not agree in general; see [Traynor 1997; Rutherford 2011].

In this article the basic fronts, $A_{m}$, will play an important role. Given $m \in \mathbb{Z}_{>0}$, $A_{m}$ is the front diagram that winds $m$ times around the annulus with $m-1$ crossings and no cusps; see Figure 1. When it is necessary to pay attention to orientations, for $m>0$, we will use $A_{m}$ (respectively $A_{-m}$ ) for the basic front oriented in the direction of the positive (respectively negative) $x$-axis.

If $\lambda=\left(\lambda_{1}, \ldots, \lambda_{\ell}\right)$ is an $\ell$-tuple of positive integers we write $A_{\lambda}=A_{\lambda_{1}} A_{\lambda_{2}} \cdots A_{\lambda_{\ell}}$ for the product of basic fronts and $A_{-\lambda}$ for the product with all orientations reversed. 


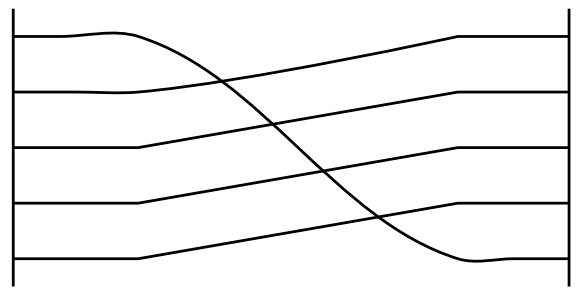

Figure 1. The basic front $A_{5}$.

2B. Kauffman polynomial in $\boldsymbol{J}^{\mathbf{1}} \boldsymbol{S}^{\mathbf{1}}$. We now describe a generalization from [Turaev 1988] of the Kauffman polynomial to smooth links (not necessarily Legendrian) in the solid torus. In practice, this invariant is computed by reducing a link diagram to products of basic fronts via skein relations. Whenever appropriate, we will view a front diagram of a Legendrian link as a smooth link diagram by placing the strand with lesser slope on top at crossings and smoothing cusps.

Let $\mathscr{D}$ denote the set of regular isotopy classes of unoriented link diagrams in the annulus. That is, we consider link diagrams up to the equivalence generated by Type II and Type III Reidemeister moves. Using the coefficient ring $R=\mathbb{Z}\left[a^{ \pm 1}, z^{ \pm 1}\right]$ we define the Kauffman skein module $\mathscr{F}$ as the quotient of the free $R$-module $R \mathscr{D}$ by the submodule generated by the Kauffman skein relations
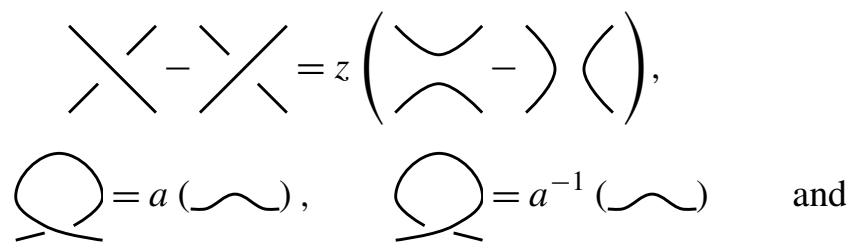

and

The product of diagrams gives a well defined product on $\mathscr{F}$ which is commutative as we now consider diagrams of smooth links rather than front diagrams of Legendrian links. Turaev [1988] showed that $\mathscr{F}$ is a polynomial $R$-algebra in the basic fronts. Thus, to a link diagram $L$ we may associate a polynomial $D_{L}\left(a, z ; A_{1}, A_{2}, \ldots\right)$ according to

$$
\mathscr{F} \cong R\left[A_{1}, A_{2}, \ldots\right], \quad[L] \leftrightarrow D_{L} .
$$

The Kauffman polynomial of an oriented link $L \subset J^{1} S^{1}$ is then defined by the normalization $F_{L}=a^{-w(L)} D_{L}$, where $w(L)$ denotes the writhe of $L$.

Chmutov and Goryunov [1997] proved that for any Legendrian link $L \subset J^{1} S^{1}$,

$$
t b(L) \leq-\operatorname{deg}_{a} F_{L} .
$$


While Chmutov and Goryunov [1997] use a different projection annulus for computing $F_{L}$, a proof of (2-4) matching our conventions for $F_{L}$ may be given precisely as in the case of the HOMFLY-PT polynomial addressed in Section 6.2 of [Rutherford 2011].

Remark 2.1. (i) Recall that a possibly empty sequence $\lambda=\left(\lambda_{1}, \ldots, \lambda_{\ell}\right)$ of positive integers is called a partition if $\lambda_{1} \geq \ldots \geq \lambda_{\ell}$. The integers $\lambda_{i}$ are called the parts of $\lambda$ and we sometimes use the notation $\lambda=1^{j_{1}} 2^{j_{2}} \ldots n^{j_{n}}$ to indicate that $\lambda$ is the partition with $j_{r}$ parts equal to $r, r=1, \ldots, n$. We note that the collection of products $A_{\lambda}$ with $\lambda$ a partition forms an $R$-module basis for $\mathscr{F}$.

(ii) The HOMFLY-PT skein module is defined in a similar manner using oriented link diagrams and an appropriate modification of the skein relations (2-1)-(2-3) (see, for instance [Rutherford 2011]). The result is a polynomial algebra generated by the oriented basic fronts [Turaev 1988]. For a given oriented link $L \subset J^{1} S^{1}$ we denote the corresponding HOMFLY-PT polynomial as

$$
P_{L} \in R\left[A_{ \pm 1}, A_{ \pm 2}, \ldots\right] .
$$

2C. Normal rulings in $J^{\mathbf{1}} S^{\mathbf{1}}$. Let $L \subset S^{1} \times \mathbb{R}$ be the front projection of a Legendrian link in the solid torus satisfying the additional assumption that all crossings and cusps have distinct $x$-coordinates none of which equals 0 . A normal ruling can be viewed locally as a decomposition of $L$ into pairs of paths. We make some notational preparation before giving the formal definition.

Denote by $\Sigma \subset S^{1}$ those $x$-coordinates which coincide with a crossing or cusp of $L$. We can write

$$
S^{1} \backslash \Sigma=\bigsqcup_{m=1}^{M} I_{m}
$$

with each $I_{m}$ an open interval (or all of $S^{1}$ if $\Sigma=\varnothing$ ). Making the convention that $I_{0}=I_{M}$, we assume that the $I_{m}$ are ordered so that $I_{m-1}$ appears immediately to the left of $I_{m}$ and $I_{M}$ contains $x=0$. On subsets of the form $I_{m} \times \mathbb{R}$ the front projection $L$ consists of some number of nonintersecting components which project homeomorphically onto $I_{m}$. We refer to these components as the strands of $L$ above $I_{m}$, and we number them from top to bottom as $1, \ldots, N(m)$. Finally, for each $m=1, \ldots, M$ we choose a point $x_{m} \in I_{m}$.

Definition 2.2. A normal ruling of the front diagram $L$ is a sequence of involutions $\rho=\left(\rho_{1}, \ldots, \rho_{M}\right)$,

$$
\rho_{m}:\{1, \ldots, N(m)\} \rightarrow\{1, \ldots, N(m)\}, \quad\left(\rho_{m}\right)^{2}=i d,
$$

satisfying the following restrictions:

(1) Each $\rho_{m}$ is fixed-point-free. 
(2) If the strands above $I_{m}$ labeled $k$ and $k+1$ meet at a left cusp in the interval $\left(x_{m-1}, x_{m}\right)$, then $\rho_{m}(k)=k+1$ and when $n \notin\{k, k+1\}$,

$$
\rho_{m}(n)= \begin{cases}\rho_{m-1}(n) & \text { if } n<k \\ \rho_{m-1}(n-2) & \text { if } n>k+1 .\end{cases}
$$

(3) A condition symmetric to (2) at right cusps.

(4) If strands above $I_{m}$ labeled $k$ and $k+1$ meet at a crossing on the interval $\left(x_{m-1}, x_{m}\right)$, then $\rho_{m-1}(k) \neq k+1$ and either

(a) $\rho_{m}=(k k+1) \circ \rho_{m-1} \circ(k k+1)$, where $(k k+1)$ denotes the transposition, or

(b) $\rho_{m}=\rho_{m-1}$.

In the second case we refer to the crossing as a switch of $\rho$. Finally, we have a requirement at switches that is known as the normality condition.

(5) If there is a switch on the interval $\left(x_{m-1}, x_{m}\right)$ then one of the following three orderings holds:

$$
\begin{aligned}
& \rho_{m}(k+1)<\rho_{m}(k)<k<k+1, \\
& \rho_{m}(k)<k<k+1<\rho_{m}(k+1), \\
& k<k+1<\rho_{m}(k+1)<\rho_{m}(k) .
\end{aligned}
$$

Remark 2.3. This definition is a slight variation on those found elsewhere in the literature. Letting $\pi: S^{1} \times \mathbb{R} \rightarrow S^{1}$ denote the projection, Chekanov and Pushkar defined a normal ruling as a continuous, fixed-point-free involution of $L \backslash \pi^{-1}(\Sigma)$ that preserves the $x$-coordinate and is subject to some requirements for continuous extension near crossings or cusps as well as a normality condition at switches. Such an involution is recovered from our definition by viewing the set $\{1,2, \ldots, N(m)\}$ that $\rho_{m}$ permutes as the set of strands above $I_{m}$.

From this perspective, the fixed-point-free condition causes the $\rho_{m}$ to divide the strands above $I_{m}$ into pairs, and in our figures we will present normal rulings by indicating this pairing. Beginning at $x=0$ and working to the right, one may cover the front diagram with pairs of continuous paths with monotonically increasing $x$-coordinates, so that a given pair of paths corresponds to strands paired by the involutions. If a path proceeds all the way around the annulus, then it will not necessarily end up where it started. However, the division of the front diagram into pairs of points at $x=0$ and $x=1$ should match up.

Paired paths are only allowed to meet at common cusp endpoints. In particular, at any crossing the two paths of the ruling that meet should belong to different pairs and, for values of $x$ near the crossing, each will have a "companion path" located somewhere above or below the crossing. The two paths can either follow the link diagram and cross each other (this corresponds to (4) (a) above) or they may switch 

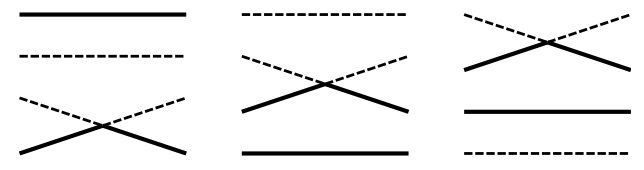

Figure 2. The normality condition.

strands by each turning a corner at the crossing. The normality condition provides a restriction on the location of the companion paths near a switch; out of six possible configurations for the switching strands and their companion strands only three are allowed. See Figure 2 for the normality condition and the right half of Figure 4 for an example of a normal ruling.

2D. Maslov potentials and graded normal rulings. Further grading restrictions may be placed on a normal ruling after the introduction of a Maslov potential for $L$. Let $p$ be a divisor of $2 r\left(L_{i}\right)$ for each component $L_{i}$ of a Legendrian link $L$. A $\mathbb{Z} / p$-valued Maslov potential $\mu$ for $L$ is a function from $L$ to $\mathbb{Z} / p$ that is constant except at cusp points, where it increases by 1 when moving from the lower strand to the upper strand. Note that a chosen orientation provides $L$ with a $\mathbb{Z} / 2$-valued Maslov potential by following the convention that strands oriented to the right (respectively left) are assigned the value 0 (respectively 1 ) mod 2.

We say that a normal ruling $\rho$ is $p$-graded with respect to a $\mathbb{Z} / p$-valued Maslov potential $\mu$ if whenever two strands $S_{1}$ and $S_{2}$ of $L$ are paired by one of the $\rho_{m}$ with $S_{1}$ above $S_{2}$ we have $\mu\left(S_{1}\right)=\mu\left(S_{2}\right)+1$.

2E. Ruling polynomials. Suppose $\mu$ is a $\mathbb{Z} / p$-valued Maslov potential for a Legendrian link $L$. The $p$-graded ruling polynomial of $L$ with respect to $\mu$ is

$$
R_{(L, \mu)}^{p}(z)=\sum_{\rho} z^{j(\rho)},
$$

where the sum is over all normal rulings of $L$ that are $p$-graded with respect to $\mu$ and

$$
j(\rho)=\# \text { switches }-\# \text { right cusps. }
$$

The ruling polynomial does not depend on the choice of Maslov potential when $p=1 ; p=2$ and $L$ is oriented; or $L$ is connected. In any of these cases we denote the ruling polynomial simply as $R_{L}^{p}$. The ruling polynomials are Legendrian isotopy invariants [Chekanov and Pushkar 2005].

2F. Generalized normal rulings. In the following definition we relax the requirements from Definition 2.2 in a manner appropriate for Theorem 1.2 to hold.

Definition 2.4. A generalized normal ruling consists of a sequence of involutions $\rho=\left(\rho_{1}, \ldots, \rho_{M}\right)$ as in Definition 2.2 subject to the following modifications: 
(1) We remove the requirement that the $\rho_{m}$ be fixed-point-free.

(2) If a crossing occurs in the interval $\left(x_{m-1}, x_{m}\right)$ between the $k$ and $k+1$ strands above $I_{m-1}$ with exactly one of these two strands a fixed point of $\rho_{m}$, then we decide if the crossing is a switch precisely as in (4) of Definition 2.2. If the crossing is indeed a switch then we require the additional normality condition that either

$$
\rho_{m}(k)=k<k+1<\rho_{m}(k+1) \quad \text { or } \quad \rho_{m}(k)<k<k+1=\rho_{m}(k+1) .
$$

(See Figure 3.)

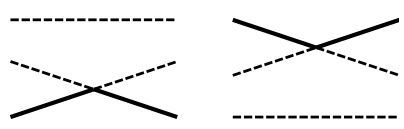

Figure 3. The normality condition for generalized rulings: The strand pictured in bold is a fixed point of $\rho_{m}$.

Remark 2.5. (i) If a crossing involving the $k$ and $k+1$ strands occurs on $\left(x_{m-1}, x_{m}\right)$ with both of the crossing strands fixed by the ruling, that is, $\rho_{m-1}(k)=k$ and $\rho_{m-1}(k+1)=k+1$, then $\rho_{m-1}=(k k+1) \circ \rho_{m-1} \circ(k k+1)$. Consequently, we will not consider such crossings to be switches.

(ii) In the presence of an appropriate Maslov potential, we can consider $p$-graded generalized normal rulings precisely as in Section 2D.

(iii) The number of generalized normal rulings of a Legendrian link is not invariant under Legendrian isotopy. However, in view of Lemma 2.6 below, the polynomials $R_{L \cdot A_{\lambda}}^{p}$ serve as some form of substitute for a "generalized ruling polynomial".

For establishing (1) of Theorem 1.2 we will use the following equivalent characterization of front diagrams that admit generalized rulings.

Lemma 2.6. A front diagram $L$ has a 1-graded (respectively 2-graded) generalized normal ruling if and only if there exists partitions $\lambda$ and $\mu$ so that $R_{L \cdot A_{\lambda}}^{1}(z) \neq 0$ (respectively $R_{L \cdot A_{\lambda} A_{-\mu}}^{2}(z) \neq 0$ ).

Proof. For simplicity, we treat the 1 -graded case first. If $R_{L \cdot A_{\lambda}}^{1}(z) \neq 0$, then the diagram $L \cdot A_{\lambda}$ has a normal ruling, $\rho$. This produces a generalized normal ruling of $L$ by restricting $\rho$ to $L$ and treating any strands of $L$ which are paired with $A_{\lambda}$ as fixed point strands. The normality condition from Definition 2.4 follows from that of Definition 2.2.

Now suppose that $L$ has a generalized normal ruling. If one of the $\rho_{m}$ has a fixed point strand, then we can continuously follow the fixed point strand around the diagram turning corners only at switches. The result is a portion of the front diagram, $C_{i}$, without cusps that we suppose winds $\lambda_{i}$ times around the annulus. 
There may be several fixed point components of this type. We may assume the $\lambda_{i}$ are ordered so that they form a partition, $\lambda$. The product $L \cdot A_{\lambda}$ has a normal ruling where each $C_{i}$ is paired with the component $A_{\lambda_{i}}$ of $\lambda$. Such a ruling is completely determined once we specify the pairing between $C_{i}$ and $A_{\lambda_{i}}$ at a single point of $C_{i}$. Now, the normality condition of Definition 2.2 follows from that of Definition 2.4, and the ordering of the factors of $A_{\lambda}$ is not important here since we do not have switches between any of the $C_{i}$; see Remark 2.5 and Figure 4 .

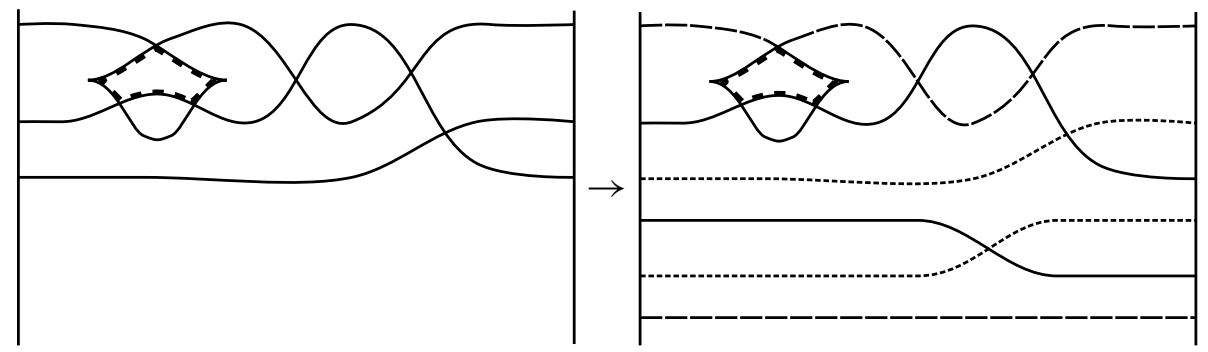

Figure 4. A generalized ruling with three fixed point strands producing a normal ruling of $L \cdot A_{\lambda}$ with $\lambda=(2,1)$.

For the 2-graded case, observe that in a 2-graded ruling the orientation of strands meeting at a switch must agree. Therefore, the $C_{i}$ each have a consistent orientation, and we choose an orientation on the component $A_{\lambda_{i}}$ accordingly.

\section{Kauffman polynomial and computation of 1-graded ruling polynomials}

An analysis of how to compute 2-graded ruling polynomials of Legendrian solid torus links from the HOMFLY-PT polynomial is done in [Rutherford 2011]. In this section, we will perform a similar analysis of the 1-graded case. We will derive formulas for the 1-graded ruling polynomial of $A_{\lambda}$, and then relate the general case to a coefficient of an appropriate specialization of the Kauffman polynomial.

3A. Normal rulings of the product $\boldsymbol{A}_{\lambda}$. Given a front diagram $L$ with normal ruling $\rho$ we define the decomposition of $L$ with respect to $\rho$ as the Legendrian link $L_{\rho}$ obtained by resolving the switches of $L$ into parallel horizontal strands as

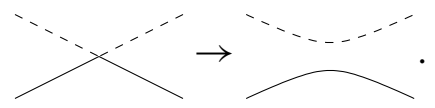

The involutions of the strands of $L$ piece together to provide an involution, which we also denote as $\rho$, now defined on all of $L_{\rho}$. The involution $\rho$ is continuous where we now view $L_{\rho}$ as a subset of $J^{1} S^{1}$ rather than just a front diagram, and its only fixed points correspond to the cusps of the front projection of $L_{\rho}$. (Compare 
with Remark 2.3.) The normal ruling of $L$ induces a normal ruling of $L_{\rho}$ where none of the crossings are switches.

We record some observations about normal rulings of the products $A_{\lambda}$.

Lemma 3.1. Suppose $\rho$ is a normal ruling of $L=A_{\lambda}$.

(1) The decomposition, $L_{\rho}$, is also a product of basic fronts.

(2) The involution $\rho$ must take a component of $L_{\rho}$ isotopic to $A_{m}$ to another component isotopic to $A_{m}$.

(3) If components $C_{1}$ and $C_{2}$ of $L_{\rho}$ share a common switch of $L$, with $C_{1}$ above $C_{2}$ on the z-axis, then the vertical ordering of the four components $C_{1}, C_{2}, \rho\left(C_{1}\right)$, and $\rho\left(C_{2}\right)$ must be one of

$$
\begin{aligned}
& {\left[\rho\left(C_{2}\right), \ldots, \rho\left(C_{1}\right), \ldots, C_{1}, C_{2}\right],} \\
& {\left[\rho\left(C_{1}\right), \ldots, C_{1}, C_{2}, \ldots, \rho\left(C_{2}\right)\right],} \\
& {\left[C_{1}, C_{2}, \ldots, \rho\left(C_{2}\right), \ldots, \rho\left(C_{1}\right)\right] .}
\end{aligned}
$$

(4) The restriction of $\rho$ to a pair of components of $L_{\rho}, C_{1}$ and $C_{2}=\rho\left(C_{1}\right)$, is completely determined by its value at a single point, $w \in C_{1}$. Moreover, if $C_{1} \cong A_{m}$ then there are precisely $m$ choices for $\rho(w) \in C_{2}$, and any one of them extends continuously to all of $C_{1}$.

(5) Two components of $L_{\rho}$ of the form $C_{1}$ and $\rho\left(C_{1}\right)$ cannot correspond to subsets of the same component of $L$.

Proof. Item (1) is clear; (2) follows from continuity of $\rho$; and (3) is a consequence of the normality condition. The first assertion of (4) follows from continuity of $\rho$. The second follows since $\rho(w)$ and $w$ must have the same $x$-coordinate and $C_{2}$ also consists of $m$ strands. That any such choice of $\rho(w)$ extends to all of $C_{1}$ is easily seen.

We prove (5) by contradiction. Suppose $C_{1}$ and $\rho\left(C_{1}\right)$ did come from the same component of $L$, and without loss of generality assume $\rho\left(C_{1}\right)$ is below $C_{1}$. They cannot meet at a switch as this would violate the normality condition. Thus, there is some other component $C_{2}$ on the other end of the switch below $C_{1}$. The only possible position of $\rho\left(C_{2}\right)$ is then between $C_{2}$ and $\rho\left(C_{1}\right)$. Then $C_{2}$ and $\rho\left(C_{2}\right)$ also came from the same component of $L$. They cannot meet at a switch, so there is some further component $C_{3}$ immediately below $C_{2}$, which is paired with a component $\rho\left(C_{3}\right)$ between $C_{3}$ and $\rho\left(C_{2}\right)$. We can continue this argument to produce arbitrarily many components of $L_{\rho}$ between $C_{1}$ and $\rho\left(C_{1}\right)$.

3B. Computing $\boldsymbol{R}_{\boldsymbol{A}_{m} \boldsymbol{A}_{m}}^{\mathbf{1}}$. The results in the previous section are sufficient to compute the ruling polynomial for the simplest possible product, $A_{m} A_{m}$ (the ruling polynomial of a single basic front $A_{m}$ is 0 by (5) of Lemma 3.1). Although this 
agrees with $R_{A_{m} A_{-m}}^{2}$ which is computed in Lemma 4.1 of [Rutherford 2011], the form of the answer given here is simplified and the proof is quite different.

Lemma 3.2. The ruling polynomial of $L=A_{m} A_{m}$ is

$$
\sum_{k=0}^{m-1}\left(\begin{array}{c}
m+k \\
2 k+1
\end{array}\right) z^{2 k}
$$

Proof. Normal rulings of $A_{m} A_{m}$ with $2 k$ switches are in bijection with subdivisions of $m$ ordered objects into $k+1$ consecutive parts, with a marked object chosen in each part.

The subdivision corresponds to choosing the location of $k$ switches within the first $A_{m}$ factor. Specifically, dividing $m$ into parts $\left(\lambda_{1}, \ldots, \lambda_{k+1}\right)$ corresponds to choosing $k$ switches so that in the decomposition, $L_{\rho}$, the first $A_{m}$ factor becomes $A_{\lambda_{1}} \ldots A_{\lambda_{k+1}}$. In $L_{\rho}$, the $A_{\lambda_{i}}$ must be paired with $k+1$ components of the same size from the second $A_{m}$ factor, by parts (2) and (5) of Lemma 3.1. Then, Lemma 3.1(3) determines the order of the components: they must be in the reverse order of the components from the first factor. The total number of switches is $2 k$.

The choice of marked object within a part $\lambda_{i}$ corresponds to choosing which strand within the $A_{\lambda_{i}}$ component is paired with the top strand of $\rho\left(A_{\lambda_{i}}\right)$ at $x=0$. These choices may be arbitrary, and they uniquely determine a ruling by part (4) of Lemma 3.1. See Figure 5.
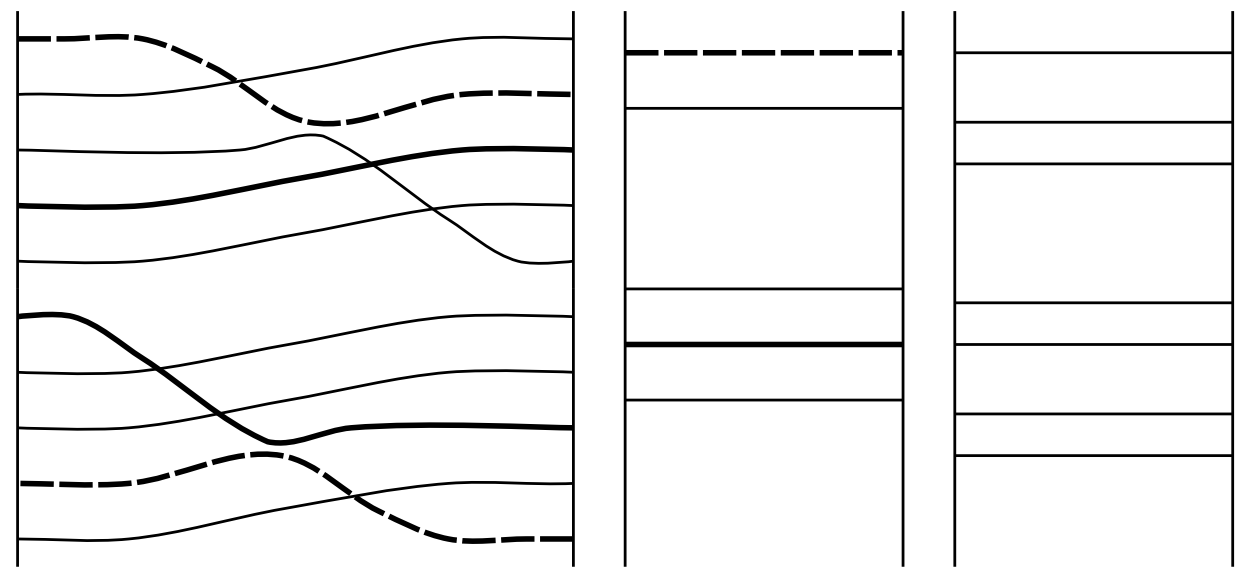

Figure 5. The bijection between rulings of $A_{5} A_{5}$ with 2 switches, divisions of 5 objects into 2 parts with a marked object in each part, and compositions of 7 into 4 positive parts.

To complete the proof, observe that subdivisions of this type are in bijection with compositions of $m+(k+1)$ into $2(k+1)$ positive parts $\left(a_{1}, b_{1}, \ldots, a_{k+1}, b_{k+1}\right)$ : two consecutive parts of size $a_{i}$ and $b_{i}$ correspond to a part $\lambda_{i}=a_{i}+b_{i}-1$ with 
the $a_{i}$-th object marked in a subdivision of $m$. The number of ways to decompose $m+k+1$ objects into $2(k+1)$ parts of positive size is well-known to be

$$
\left(\begin{array}{c}
(m+k+1)-1 \\
2(k+1)-1
\end{array}\right)=\left(\begin{array}{c}
m+k \\
2 k+1
\end{array}\right) .
$$

This gives us the sum for the ruling polynomial.

This formula will be used in the next section, so we will write $\langle m\rangle$ for the ruling polynomial $R_{A_{m} A_{m}}(z)$, following the convention in [Rutherford 2011].

3C. A formula for arbitrary products of basic fronts. We will use the formula for $\langle m\rangle$ to calculate the ruling polynomial of $A_{\lambda}$ for an arbitrary $\lambda$.

Given a normal ruling $\rho$ of $L=A_{\lambda}$, define the block $B_{i j}$ to consist of those components of the decomposition $L_{\rho}$ which originated in the $i$-th component of $L$, and are paired by $\rho$ with components that originated in the $j$-th component of $L$. The size of the block, $b_{i j}$, is the number of points in $B_{i j}$ with some fixed $x$-coordinate, away from crossings.

Lemma 3.3. Given a normal ruling of

$$
L=A_{\lambda}=A_{\lambda_{1}} A_{\lambda_{2}} \ldots A_{\lambda_{n}},
$$

the blocks in the $i$-th component of $L$ consist of vertically consecutive components of $L_{\rho}$, and are themselves vertically ordered as

$$
B_{i, i-1} B_{i, i-2} \ldots B_{i, 1} B_{i, n} B_{i, n-1} \ldots B_{i, i+1},
$$

where some blocks may be empty.

Proof. Suppose that when we resolve $A_{\lambda_{i}}$ at switches, we get the components $C_{1}, C_{2}, \ldots, C_{k}$, in that vertical order. If, for some $j, \rho\left(C_{j}\right)$ is above $C_{j}$, then the normality condition demands that $\rho\left(C_{j-1}\right)$ is between $\rho\left(C_{j}\right)$ and $C_{j-1}$. Similarly, if $\rho\left(C_{j}\right)$ is below $C_{j}$, then $\rho\left(C_{j+1}\right)$ must be between $C_{j+1}$ and $\rho\left(C_{j}\right)$.

As a result, if $\rho\left(C_{j_{1}}\right)$ and $\rho\left(C_{j_{2}}\right)$ come from the same component of $L$, then $\rho\left(C_{j}\right)$ for $j_{1} \leq j \leq j_{2}$ are between $\rho\left(C_{j_{1}}\right)$ and $\rho\left(C_{j_{2}}\right)$. This implies each block is made up of some number of consecutive components. And due to the normality condition, the ordering of any two consecutive blocks must be either $B_{i, j+1} B_{i, j}$, with $j>i$, or $B_{i, j-1} B_{i, j}$, with $j<i$ (with the caveat that some of the blocks may be empty, if $\rho$ does not pair two components of $L$ at all). Putting this together yields the block ordering above.

This means that once we pick the sizes of the blocks $b_{i, 1}, \ldots, b_{i, n}$, the locations of the blocks are determined. To complete the calculation of the ruling polynomial, observe that the choice of a normal ruling of the blocks $B_{i j}$ and $B_{j i}$, with sizes $b_{i j}=b_{j i}=m$, is equivalent to the choice of a normal ruling of $A_{m} A_{m}$. 
Theorem 3.4. Let $\langle m\rangle$ denote the ruling polynomial of $A_{m} A_{m}$, with $\langle 0\rangle$ taken to be $z^{-2}$. Then the ruling polynomial of $A_{\lambda}=A_{\lambda_{1}} A_{\lambda_{2}} \ldots A_{\lambda_{n}}$ is given by

$$
z^{n(n-2)} \sum_{\left(b_{i j}\right) \in M_{\lambda}} \prod_{i<j}\left\langle b_{i j}\right\rangle
$$

where $M_{\lambda}$ is the set of all symmetric matrices $\left(b_{i j}\right)$ with nonnegative integer entries such that the row sums $\sum_{j=1}^{n} b_{i j}=\lambda_{i}$ and the trace $\operatorname{tr}\left(b_{i j}\right)=0$.

Proof. The choice of a matrix in $M_{\lambda}$ is equivalent to the choice of block sizes $b_{i j}$. By Lemma 3.3, this also fixes the locations of the blocks. A normal ruling of $A_{\lambda}$ is then completely determined by its restriction to pairs of blocks $B_{i j}$ and $B_{j i}$.

If the block size $b_{i j}$ is nonzero, then $\left\langle b_{i j}\right\rangle$ describes the possible restrictions of the normal rulings to the union $B_{i j} \cup B_{j i}$. We take the product to combine these normal rulings, but we have to account for the switches between the blocks. If all block sizes are nonzero, then there will be $n-2$ switches in each of the $n$ components of $L$, giving us a factor of $z^{n(n-2)}$. Any block $B_{i j}$ of size 0 will reduce this number by 1 in component $j$, but the corresponding block $B_{j i}$ will reduce the number of switches by 1 in component $i$; this gives a factor of $z^{-2}$ which is accounted for by the convention of $\langle 0\rangle=z^{-2}$.

Corollary 3.5. The 1-graded ruling polynomial is commutative in front diagram products: that is, the ruling polynomials of

$$
A_{\lambda_{1}} A_{\lambda_{2}} \ldots A_{\lambda_{i}} A_{\lambda_{i+1}} \ldots A_{\lambda_{n}} \quad \text { and } \quad A_{\lambda_{1}} A_{\lambda_{2}} \ldots A_{\lambda_{i+1}} A_{\lambda_{i}} \ldots A_{\lambda_{n}}
$$

are equal.

Proof. There is an easy bijection between the possibilities for the matrix $M_{\lambda}$ and the new matrix $M_{\lambda^{\prime}}$ : we simply exchange the $i$-th and $(i+1)$-th columns and rows; the summands $\prod_{i<j}\left\langle b_{i j}\right\rangle$ do not change.

3D. Calculating the ruling polynomial from the Kauffman polynomial. In $\mathbb{R}^{3}$, the 1-graded and 2-graded ruling polynomial of arbitrary Legendrian links may be easily recovered from the Kauffman and HOMFLY-PT polynomials. The second author shows in [Rutherford 2011] that the 1-graded (respectively 2-graded) ruling polynomial of a link $L$ is the coefficient of $a^{-t b(L)}$ in the Kauffman polynomial (respectively HOMFLY-PT polynomial) of $L$. In the case of Legendrian solid torus links we first need to specialize the extra variables in a nonmultiplicative manner.

Using the notation of Section $2 \mathrm{~B}$, we consider the $R$-module homomorphism $\Psi: \mathscr{F} \cong R\left[A_{1}, A_{2}, \ldots\right] \rightarrow R$ determined by $A_{\lambda} \mapsto R_{A_{\lambda}}^{1}(z)$ when $\lambda$ is a partition. (Compare with Remark 2.1.) Given a link diagram $L$, we let $\widehat{D}_{L}(a, z)=\Psi\left(D_{L}\right)$, and $\widehat{F}_{L}(a, z)=a^{-w(L)} \widehat{D}_{L}(a, z)$. 
Theorem 3.6. Let $L \subset J^{1} S^{1}$ be any Legendrian solid torus link. Then the 1-graded ruling polynomial $R_{L}^{1}(z)$ is equal to the coefficient of $a^{-t b(L)}$ in $\widehat{F}_{L}(a, z)$.

This result is analogous to Theorem 6.3 of [Rutherford 2011], where it is shown that we can recover the 2-graded ruling polynomial from such a specialization of the HOMFLY-PT polynomial. The proof, via induction on a certain measure of complexity of a front diagram, carries through in the 1-graded case as well. The base case consists of all products of basic fronts where the result follows from the crucial Corollary 3.5. Next, it is observed that the ruling polynomial and the coefficient of $a^{-t b(L)}$ in $\widehat{F}_{L}$ share common skein relations that are Legendrian analogs of equations (2-1)-(2-3) (see [Rutherford 2006; Rutherford 2011]). Then, just as in [Rutherford 2011], the inductive step is completed by an algorithm which uses these skein relations to evaluate the invariants in terms of front diagrams of lesser complexity.

Example. Consider the Legendrian knots $L_{1}$ and $L_{2}=L_{1} \cdot A_{2} A_{1}$ pictured in Figure 4, and suppose orientations are chosen so that all strands are oriented to the right when they pass through the vertical line $x=0$. The Kauffman polynomials are given by

$$
\begin{aligned}
& F_{L_{1}}=A_{1} \times[\left.a^{-1}\left(-z-z^{3}\right)+a^{-2} z^{4}+a^{-3}\left(z+2 z^{3}\right)+a^{-4} z^{2}\right] \\
&+A_{3} \times\left[a^{-1}\left(z+z^{3}\right)+a^{-2}\left(-z^{2}-z^{4}\right)+a^{-3}\left(-z-z^{3}\right)\right] \\
&+A_{2} A_{1} \times\left[a^{-1}\left(1+z^{2}\right)-a^{-2} z^{3}-a^{-3} z^{2}\right],
\end{aligned}
$$

and $F_{L_{2}}=a^{-1} A_{2} A_{1} F_{L_{1}}$. We have $t b\left(L_{1}\right)=1$ and $t b\left(L_{2}\right)=2$, so in both cases the estimate (2-4) is sharp.

Theorem 3.4 gives $R_{A_{(2,1,1)}}^{1}(z)=z, R_{A_{(3,2,1)}}^{1}(z)=2 z+z^{3}$, and $R_{A_{(2,2,1,1)}}^{1}(z)=$ $2+3 z^{2}$. This allows us to compute

$\widehat{F}_{L_{2}}=a^{-2}\left(2+6 z^{2}+5 z^{4}+z^{6}\right)+a^{-3}\left(-4 z^{3}-5 z^{5}-z^{7}\right)+a^{-4}\left(-3 z^{2}-4 z^{4}-z^{6}\right)+a^{-5} z^{3}$, and Theorem 3.6 gives $R_{L_{2}}^{1}(z)=2+6 z^{2}+5 z^{4}+z^{6}$, which can be verified directly.

\section{Generalized normal rulings and the Thurston-Bennequin estimates}

In this section we establish the equivalence (1) of Theorem 1.2 which follows from Lemma 2.6 together with the following:

Theorem 4.1. Let $L$ be a Legendrian link in the solid torus. Then the equality

$$
\operatorname{tb}(L)=-\operatorname{deg}_{a} F_{L}
$$

holds if and only if there exists a partition $\lambda$ so that $L \cdot A_{\lambda}$ has a normal ruling. 
Proof of Theorem 4.1. One direction is straightforward. Suppose that, for some $\lambda$, $L^{\prime}=L \cdot A_{\lambda}$ has a normal ruling. Then the ruling polynomial of $L^{\prime}$ is nontrivial, so the coefficient of $a^{-t b(L)}$ is nonzero. Therefore $t b\left(L^{\prime}\right) \geq-\operatorname{deg}_{a} F_{L^{\prime}}$ which, combined with the inequality (2-4), gives us an equality $t b\left(L^{\prime}\right)=-\operatorname{deg}_{a} F_{L^{\prime}}$. However, $t b\left(L^{\prime}\right)=t b\left(L \cdot A_{\lambda}\right)=t b(L)+w\left(A_{\lambda}\right)$, since $A_{\lambda}$ has no cusps. In addition, $D_{L^{\prime}}=A_{\lambda} \cdot D_{L}$, so $F_{L^{\prime}}=a^{-w\left(A_{\lambda}\right)} A_{\lambda} \cdot F_{L}$, and we compute

$$
-\operatorname{deg}_{a} F_{L}=-w\left(A_{\lambda}\right)-\operatorname{deg}_{a}\left(F_{L^{\prime}}\right)=-w\left(A_{\lambda}\right)+t b\left(L^{\prime}\right)=t b(L) .
$$

Now suppose $t b(L)=-\operatorname{deg}_{a} F_{L}$. We will find a $\lambda$ such that $L \cdot A_{\lambda}$ has a normal ruling.

Let $\sum_{\mu} p_{\mu}(z) A_{\mu}$ be the coefficient of $a^{-t b(L)}$ in $F_{L}$, where the $p_{\mu}(z)$ are polynomials in $z$ and $z^{-1}$. This coefficient is nonzero, or else the degree equality would not hold, so $p_{\mu}(z) \neq 0$ for at least one $\mu$. Let $k$ be the smallest integer such that at least one $p_{\mu}$ has a nonzero coefficient of $z^{k}$.

By Theorem 3.6, the ruling polynomial of $L \cdot A_{\lambda}$ is

$$
\sum_{\mu} p_{\mu}(z) R_{A_{\mu} A_{\lambda}}(z)
$$

We will prove that for some $\lambda$, this polynomial is nonzero (and therefore a normal ruling exists) by looking at the $z^{k}$ coefficient of this polynomial. Since $R_{A_{\mu} A_{\lambda}}(z)$ is a polynomial in $z$ with no terms of $z^{-1}$ or lower degree, the only way to get a $z^{k}$ coefficient is from the product of $p_{\mu}(z)\left[z^{k}\right]$ and $R_{A_{\mu} A_{\lambda}}(z)\left[z^{0}\right]$ for some $\mu$ (here, $f(z)\left[z^{i}\right]$ denotes the coefficient of $z^{i}$ in $f(z)$ ). Denote $p_{\mu}(z)\left[z^{k}\right]$ by $a_{\mu}$, and $R_{A_{\mu}}(z)\left[z^{0}\right]$ (which is the number of switchless rulings of $A_{\mu}$ ) by $C(\mu)$.

The quantity $C(\mu)$ is easy to calculate. Without switches, each component of size $k$ must simply be paired with another component of size $k$ in one of $k$ ways. In particular, this is only possible if there is an even number of each component size. Define the double factorial by

$$
(2 k-1) ! !=(2 k-1)(2 k-3)(\cdots)(3)(1)=(2 k) ! /\left(2^{k} k !\right) .
$$

It counts the number of ways to divide $2 k$ objects into pairs. It is clear that

$$
C(\mu)= \begin{cases}\prod_{k=1}^{n} k^{a_{k}}\left(2 a_{k}-1\right) ! ! & \text { if } \mu=1^{2 a_{1}} 2^{2 a_{2}} \ldots n^{2 a_{n}}, \\ 0 & \text { else. }\end{cases}
$$

We wish to prove that for some $\lambda, \sum_{\mu} a_{\mu} C(\mu \cdot \lambda) \neq 0$. Here, if

$$
\mu=1^{a_{1}} 2^{a_{2}} \ldots n^{a_{n}} \text { and } \lambda=1^{b_{1}} 2^{b_{2}} \ldots n^{b_{n}},
$$

we will denote by $\mu \cdot \lambda$ the partition

$$
1^{a_{1}+b_{1}} 2^{a_{2}+b_{2}} \ldots n^{a_{n}+b_{n}} .
$$


Let $M$ be the collection of all partitions such that:

(1) The parts of the partition are all no larger than $n$, for some $n$.

(2) Parts of each size occur between 0 and $2 m-1$ times, for some $m$.

We choose the parameters $m$ and $n$ such that we include all partitions $\mu$ with $a_{\mu} \neq 0$.

Let $V$ be a $n^{2 m}$-dimensional real vector space with basis vectors $e_{\lambda}$ for $\lambda \in M$. For each $\mu \in M$, consider the vectors

$$
v_{\mu}=\sum_{\lambda \in M} C(\mu \cdot \lambda) e_{\lambda}
$$

in $V$. We will show that these vectors also form a basis of $V$, and are therefore linearly independent. From there, observe that

$$
\sum_{\lambda \in M}\left(\sum_{\mu \in M} a_{\mu} C(\mu \cdot \lambda)\right) e_{\lambda}=\sum_{\mu \in M} a_{\mu}\left(\sum_{\lambda \in M} C(\mu \cdot \lambda) e_{\lambda}\right)=\sum_{\mu \in M} a_{\mu} v_{\mu} .
$$

If the coefficients $a_{\mu}$ on the right are not all 0 , then because the $v_{\mu}$ are linearly independent the resulting sum is a nonzero vector of $V$. Therefore the coefficients in terms of $e_{\lambda}$ are not all 0 as well - that is, for some $\lambda, \sum_{\mu} a_{\mu} C(\mu \cdot \lambda) \neq 0$. So once we have the result of linear independence, we are done.

From the formula for $C(\mu)$, it's easy to calculate that $C(\mu \cdot \lambda)$ can be written as a product of $C\left(k^{a_{k}} \cdot k^{b_{k}}\right)$, over all $k$, where $a_{k}$ and $b_{k}$ are the number of parts of size $k$ in $\lambda$ and $\mu$ respectively. Suppose we write $V$ as the tensor product $\bigotimes_{i=1}^{n} \mathbb{R}^{2 m}$, identifying the basis vector $e_{j_{1}} \otimes e_{j_{2}} \otimes \cdots \otimes e_{j_{n}}$ on the left with the basis vector $e_{\lambda}$ on the right, where $\lambda=1^{j_{1}} 2^{j_{2}} \ldots n^{j_{n}}$. Here we use a slightly nonstandard basis of $\mathbb{R}^{2 m}:$ it is 0 -indexed and consists of $\left\{e_{0}, e_{1}, \ldots, e_{2 m-1}\right\}$, for ease of notation.

Then, if $\mu=1^{a_{1}} 2^{a_{2}} \ldots n^{a_{n}}$,

$$
\begin{aligned}
v_{\mu}=\sum_{\lambda \in M} C(\mu \cdot \lambda) e_{\lambda} & =\sum_{1^{b_{1} \ldots n^{b_{n}} \in M}}\left(\prod_{i=1}^{n} C\left(i^{a_{i}} \cdot i^{b_{i}}\right)\right)\left(\bigotimes_{i=1}^{n} e_{b_{i}}\right) \\
& =\sum_{1^{b_{1} \ldots n^{b_{n}} \in M}}\left(\bigotimes_{i=1}^{n} C\left(i^{a_{i}} \cdot i^{b_{i}}\right) e_{b_{i}}\right)=\bigotimes_{i=1}^{n}\left(\sum_{j=0}^{2 m-1} C\left(i^{a_{i}} \cdot i^{j}\right) e_{j}\right) .
\end{aligned}
$$

Therefore, rather than prove that the vectors $v_{\mu}$ are a basis of $V$, it suffices to prove that the vectors $u_{k}=\sum_{j=0}^{2 m-1} C\left(i^{k} \cdot i^{j}\right) e_{j}$, as $k$ goes from 0 to $2 m-1$, are a basis of $\mathbb{R}^{2 m}$. There are three simplifying observations to be made:

(1) $C\left(i^{k} \cdot i^{j}\right)=0$ if $k \not \equiv j(\bmod 2)$. Therefore $u_{k}$ is a linear combination only of the odd-indexed $e_{j}$ if $k$ is odd, and only of the even-indexed $e_{j}$ if $k$ is even. Furthermore, $C\left(i^{k} \cdot i^{j}\right)=C\left(i^{k-1} \cdot i^{j+1}\right)$, so $u_{2 k}$ and $u_{2 k-1}$ have the same coefficients, just shifted over by one index. As a result, we will only show the independence of the vectors $u_{0}, u_{2}, \ldots, u_{2 m-2}$ - the result for $u_{1}, u_{3}, \ldots, u_{2 m-1}$ is similar. 
(2) By the first observation, we have

$$
u_{2 k}=\sum_{j=0}^{m-1} C\left(i^{2 k} \cdot i^{2 j}\right) e_{2 j}=i^{k} \sum_{j=0}^{m-1} C\left(1^{2 k} \cdot 1^{2 j}\right)\left(i^{j} e_{2 j}\right) .
$$

This corresponds to starting in the case $i=1$, then scaling both the $u_{2 k}$ and the $e_{2 j}$ by powers of $i$ - a scaling which doesn't change the question of linear independence one way or the other. Therefore it suffices to consider the case $i=1$.

(3) Finally, we can scale each $u_{2 k}$ by $C\left(1^{2 k}\right)$ (which, too, doesn't affect linear independence). Now we want to look at

$$
u_{2 k}^{\prime}=\sum_{j=0}^{m-1} C\left(1^{2 k} \cdot 1^{2 j}\right) / C\left(1^{2 k}\right) e_{2 j}=\sum_{j=0}^{m-1}\left(\prod_{\ell=1}^{j}(2 k+2 \ell-1)\right) e_{2 j} .
$$

If we put the coefficients of $u_{2 k}^{\prime}$ as columns of a matrix, (that is, $j$ indexes the rows and $k$ indexes the columns), we get

$$
\left(\begin{array}{cccc}
1 & 1 & \cdots & 1 \\
1 & 3 & \cdots & 2 m-1 \\
1 \cdot 3 & 3 \cdot 5 & \ldots & (2 m-1)(2 m+1) \\
\vdots & \vdots & \ddots & \vdots \\
1 \cdot 3 \cdots(2 m-1) & 3 \cdot 5 \cdots(2 m+1) & \ldots & (2(m-1)+1)(\cdots)(4(m-1)-1)
\end{array}\right)
$$

Here, the entries in the $j$-th row are given by $f_{j}(k)=\prod_{\ell=1}^{j}(2 k+2 \ell-1)$, which is a degree $j$ polynomial function. In particular, $f_{j}(k)$ can be written as $(2 k)^{j}$ plus lower-order terms; these lower-order terms are necessarily a linear combination of $f_{1}(k), \ldots, f_{j-1}(k)$. Therefore, we can use row operations to eliminate the lowerorder terms, so that the resulting matrix is

$$
\left(\begin{array}{cccc}
1 & 1 & \ldots & 1 \\
1 & 2 & \ldots & m \\
1 & 4 & \ldots & m^{2} \\
\vdots & \vdots & \ddots & \vdots \\
1 & 2^{m-1} & \ldots & m^{m-1}
\end{array}\right)
$$

This is a Vandermonde matrix whose determinant is $\prod_{j \neq k}(j-k) \neq 0$. Therefore the vectors $u_{2 k}^{\prime}$ (and $u_{2 k}$ ) form a basis of $\mathbb{R}^{2 m}$, which completes the proof.

4A. The 2-graded case and the HOMFLY-PT estimate. A similar approach applies in the case of the HOMFLY-PT polynomial, $P_{L}$. The proof of the reverse implication is identical. For the forward implication, we suppose $t b(L)=-\operatorname{deg}_{a} P_{L}$ and consider the coefficient of the lowest power $z^{k}$ that appears in the $a^{-t b(L)}$ term 
of $P_{L}$,

$$
\sum_{\alpha, \beta} b_{(\alpha, \beta)} A_{\alpha} A_{-\beta}
$$

Fix parameters $m$ and $n$ so that the set

$$
M=\left\{(\mu, v) \mid \mu=1^{a_{1}} \cdots n^{a_{n}}, v=1^{b_{1}} \cdots n^{b_{n}}, 1 \leq a_{i}, b_{i} \leq m\right\}
$$

contains all $(\alpha, \beta)$ such that $b_{(\alpha, \beta)} \neq 0$.

Using Theorem 6.3 in [Rutherford 2011], for any $(\mu, \nu) \in M$ the coefficient of $z^{k}$ in the 2-graded ruling polynomial of $L \cdot A_{\mu} A_{-v}$ is given by

$$
\sum_{\alpha, \beta} b_{(\alpha, \beta)} R_{A_{\alpha \cdot \mu} A_{-\beta \cdot v}}^{2}(0) \text {. }
$$

It suffices to show that the coefficient matrix

$$
A=\left(R_{A_{\alpha \cdot \mu} A_{-\beta \cdot v}}^{2}(0)\right)_{(\alpha, \beta),(\mu, \nu) \in M}
$$

is nonsingular. Writing $\alpha=1^{a_{1}} \ldots n^{a_{n}}, \beta=1^{b_{1}} \ldots n^{b_{n}}, \mu=1^{c_{1}} \ldots n^{c_{n}}$, and $v=$ $1^{d_{1}} \ldots n^{d_{n}}$, one has

$$
R_{A_{\alpha \cdot \mu} A_{-\beta \cdot v}}^{2}(0)=\prod_{k=1}^{n} \delta_{a_{k}+c_{k}, b_{k}+d_{k}} k^{a_{k}+c_{k}}\left(a_{k}+c_{k}\right) ! .
$$

Thus, $A$ is a tensor product (Kronecker product) of matrices

$$
A_{k}=\left(\delta_{a+c, b+d} k^{a+c}(a+c) !\right)_{(a, b),(c, d)} .
$$

Due to the Kronecker delta, each $A_{k}$ is a direct sum (block matrix) of matrices $B_{l}$, $l \in \mathbb{Z} \cap[-n, n]$ obtained from keeping rows and columns satisfying $a-b=d-c=l$.

The proof is completed by showing that each $B_{l}$ is nonsingular. We treat the case $l \geq 0$ as $l<0$ is similar. Then, $l \leq a, d \leq n$ and $B_{l}=\left(k^{a+d-l}(a+d-l) !\right)$. Dividing rows by $k^{a-l}$ and columns by $k^{d} \cdot d$ ! leaves

$$
\frac{(a+d-l) !}{d !}=\left(f_{a}(d)\right) \text {, }
$$

where $f_{a}(x)=\prod_{j=1}^{a-l}(j+x)$ is a polynomial of degree $a-l$. Elementary row operations reduce this to a nonsingular Vandermonde matrix.

\section{Augmentations and generalized normal rulings}

In this final section we complete the proof of Theorem 1.2 by establishing that:

For any Legendrian link $L \subset J^{1} S^{1}$ with $\mathbb{Z} / p$-graded Maslov potential, $\mu$, the following are equivalent:

(A) The Chekanov-Eliashberg algebra $(\mathscr{A}(L), d)$ admits a $p$-graded augmentation.

(B) The front projection of $L$ has a $p$-graded generalized normal ruling. 
We begin by briefly recalling the aspects of the Chekanov-Eliashberg DGA that are important for the proof. The reader is referred to [Ng and Traynor 2004] for the original, more detailed treatment of this DGA in the $J^{1} S^{1}$ setting.

Given a Legendrian knot or link $L \subset J^{1} S^{1}$, the Lagrangian projection $\pi_{x y}(L)$ of $L$ to the $x y$-annulus is an immersed curve. The Chekanov-Eliashberg DGA $(\mathscr{A}(L), d)$ is a graded algebra $\mathscr{A}(L)$ with a degree -1 differential $d$, defined via a generic Lagrangian projection of $L$.

After a small Legendrian isotopy, we may assume $\pi_{x y}(L)$ to have only finitely many transverse double points which we label as $q_{1}, \ldots, q_{n}$. Then the algebra $\mathscr{A}(L)$ is the free associative $\mathbb{Z} / 2$-algebra with unit generated by the double points $q_{1}, \ldots, q_{n}$. The set of monic noncommutative monomials in the $q_{i}$ forms a linear basis for $\mathscr{A}(L)$. If $L$ is connected, then $\mathscr{A}(L)$ has a $\mathbb{Z} / 2 r(L)$ grading. In general, the grading depends on a choice of Maslov potential for $L$. The differential $d$ is defined by counting certain immersed discs in the $x y$-annulus with boundary mapped to the Lagrangian projection of $L$.

Definition 5.1. An augmentation of $(\mathscr{A}(L), d)$ is an algebra homomorphism

$$
\varepsilon: \mathscr{A}(L) \rightarrow \mathbb{Z} / 2
$$

satisfying

(i) $\varepsilon(1)=1$, and

(ii) $\varepsilon \circ d=0$.

In addition, $\varepsilon$ is $p$-graded if $\varepsilon\left(q_{i}\right) \neq 0$ implies $\left|q_{i}\right|=0 \bmod p$.

The existence of an augmentation of $(\mathscr{A}(L), d)$ is a property that is invariant under Legendrian isotopy. This follows from the fact that the "stable tame isomorphism type" (see [Chekanov 2002; Ng and Traynor 2004]) of $(\mathscr{A}(L), d)$ is unchanged by a Legendrian isotopy. Therefore, in establishing the equivalence of (A) and (B) we may work with the Chekanov-Eliashberg algebra of a Legendrian isotopic link $L^{\prime}$. The links $L^{\prime}$ which we will consider have a standard form so that $\left(\mathscr{A}\left(L^{\prime}\right), d\right)$ may be described in a formulaic manner from the front projection of $L^{\prime}$ (and this front projection is combinatorially the same as that of $L$ ). For this reason we do not present the differential or the grading of the Chekanov-Eliashberg DGA in full generality here.

5A. The DGA of a resolved front diagram with splashes. Given a Legendrian $L \subset J^{1} S^{1}$ we begin by modifying the front diagram of $L$ via (a slight variation of) the resolution procedure of $\mathrm{Ng}$ and Traynor [2004]. Beginning near $x=0$ and working from left to right, we alter the front projection of $L$ by an isotopy in the $x z$-annulus as follows. We arrange so that, except for intervals near $x=1$ or immediately prior to a crossing or right cusp, the slopes of the strands are constant 
and strictly decreasing as we move from the top to bottom. Further, we will assume that all strands usually have nonpositive slope. It is no problem to produce these conditions after a left cusp, but with crossings and right cusps the slopes of the two relevant strands will need to be interchanged prior to the crossing or cusp. As the $y$-coordinate is given by the slope $d z / d x$, this has the effect of producing double points on the Lagrangian projection corresponding to (but located to the left of) the crossings and right cusps of the front projection of $L$. Finally, when we near $x=1$ the strands have become very spread out and moved below their original $z$ values at $x=0$. Beginning with the top strand and then proceeding successively to the lowest strands, we return each strand back to its initial position via a steep upward step. This creates several new crossings on the Lagrangian projection; see Figure 6.
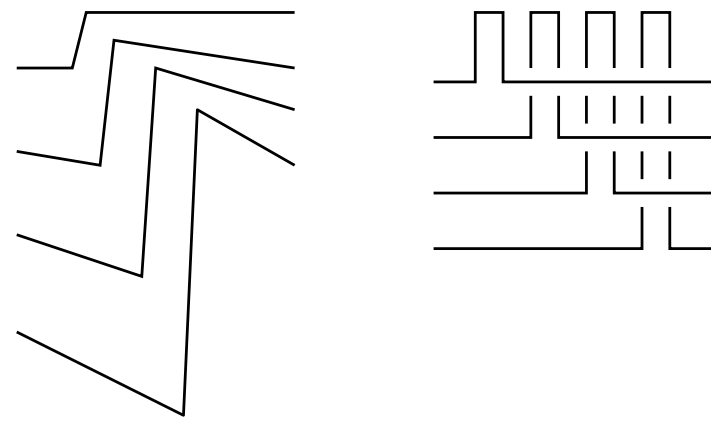

Figure 6. The front projection (left) and Lagrangian projection (right) of $L^{\prime}$ in an interval immediately to the left of $x=1$.

Next, we add "splashes". This is a variant of a technique introduced in [Fuchs 2003]; see Remark 5.2. We view the $S^{1}$ factor of $J^{1} S^{1}$ as $[0,1]$ with 0 and 1 identified. In notation similar to that of Section 2, let $0=x_{0}<x_{1}<\cdots<x_{M}=1$ be a partitioning of the interval $[0,1]$ such that no $x_{m}$ coincides with the $x$-coordinate of a crossing or cusp and each interval $\left(x_{m-1}, x_{m}\right)$ contains exactly one crossing or cusp. For each $m=1, \ldots, M-1$, we add a miniature version of the steps appearing in the part of the resolution procedure near $x=1$ into a small interval centered at $x_{m}$. That is, beginning at the top strand and then working downward add a brief but steep (smooth) upward step into the diagram. This has a minimal effect on the front projection but alters the Lagrangian projection at each $x_{m}$ by replacing what had been several parallel lines with a collection of crossings similar to those pictured in the right half of Figure 6. Denote the Legendrian link resulting from the combination of these two procedures as $L^{\prime}$.

We now give a complete description of the Chekanov-Eliashberg DGA of $L^{\prime}$. For each $1 \leq m \leq M$, let $N(m)$ denote the number of intersection points of $L$ with 
the plane $x=x_{m}$. The generators of $\mathscr{A}\left(L^{\prime}\right)$ come from two sources. First, we have generators corresponding to the crossings and right cusps of the front projection of $L$ via the resolution procedure. In addition, for each $1 \leq m \leq M$ we have two upper triangular matrices worth of generators, $x_{i j}^{m}$ and $y_{i j}^{m}$ with $1 \leq i<j \leq N(m)$. These correspond to the double points created by the splashes and the final step of the resolution procedure.

The grading. If $L$ is equipped with a $\mathbb{Z} / p$-graded Maslov potential, $\mu$, then $\mathscr{A}\left(L^{\prime}\right)$ is $\mathbb{Z} / p$-graded. We will describe the degree $\left|q_{i}\right| \in \mathbb{Z} / p$ assigned to the generators of $\mathscr{A}\left(L^{\prime}\right)$; degrees then extend additively as $|x \cdot y|=|x|+|y|$.

In the following, $\mu(m, i)$ denotes the value of the Maslov potential on the $i$-th strand at $x_{m}$. (As in Section 2, we label strands from top to bottom.) The generators of $\mathscr{A}\left(L^{\prime}\right)$ coming from splashes have degrees

$$
\left|x_{i j}^{m}\right|=\mu(m, i)-\mu(m, j) \quad \text { and } \quad\left|y_{i j}^{m}\right|=\mu(m, i)-\mu(m, j)-1 .
$$

In addition, a crossing $b_{m}$ between the $k$ and $k+1$ strands occurring in the interval $\left(x_{m-1}, x_{m}\right)$ has $\left|b_{m}\right|=\mu(m, k+1)-\mu(m, k)$, and all right cusps have degree 1 .

The differential. Formulas for the differential $d$ are most efficiently provided by placing the generators $x_{i j}^{m}$ and $y_{i j}^{m}$ into strictly upper triangular matrices

$$
X_{m}=\left(x_{i j}^{m}\right) \quad \text { and } \quad Y_{m}=\left(y_{i j}^{m}\right)
$$

for each $m$. (Here, $x_{i j}^{m}=y_{i j}^{m}=0$ if $i \geq j$.) As the $x$-coordinate is $S^{1}$-valued, it is important to make the convention that $X_{0}=X_{M}$ and $Y_{0}=Y_{M}$. Then, applying the differential to each entry, we have the formulas

$$
\begin{aligned}
d Y_{m} & =\left(Y_{m}\right)^{2} \quad \text { and } \\
d X_{m} & =Y_{m}\left(I+X_{m}\right)+\left(I+X_{m-1}\right) \widetilde{Y}_{m-1}
\end{aligned}
$$

with $I$ an identity matrix of the appropriate size. The precise form of $\widetilde{Y}_{m-1}$ depends on the tangle appearing on the interval $\left(x_{m-1}, x_{m}\right)$ and is described presently.

Suppose that $\left(x_{m-1}, x_{m}\right)$ contains a crossing, $b_{m}$, between the strands labeled $k$ and $k+1$. Then

$$
d b_{m}=y_{k, k+1}^{m-1} \quad \text { and } \quad \widetilde{Y}_{m-1}=B_{k, k+1} \widehat{Y}_{m-1} B_{k, k+1}^{-1},
$$

where $B_{k, k+1}$ and $B_{k, k+1}^{-1}$ agree with the identity matrix except for a $2 \times 2$ block along the diagonal in rows $k$ and $k+1$, having the form $\left[\begin{array}{cc}0 & 1 \\ 1 & b_{m}\end{array}\right]$ for $B_{k, k+1}$ and $\left[\begin{array}{cc}b_{m} & 1 \\ 1 & 0\end{array}\right]$ for $B_{k, k+1}^{-1}$, and $\widehat{Y}_{m-1}$ is the matrix $Y_{m-1}$ with 0 replacing the entry $y_{k, k+1}^{m-1}$.

Next, we suppose $\left(x_{m-1}, x_{m}\right)$ contains a single left cusp between the strands labeled $k$ and $k+1$ at $x_{m}$. Then,

$$
\widetilde{Y}_{m-1}=J_{k} Y_{m-1} J_{k}^{\mathrm{T}}+E_{k, k+1},
$$


where $J_{k}$ is the $N(m) \times N(m)$ identity matrix with columns $k$ and $k+1$ removed and $E_{k, k+1}$ is a matrix with a single nonzero entry in the $k, k+1$ position.

Finally, we suppose $\left(x_{m-1}, x_{m}\right)$ contains a single right cusp, $c_{m}$, between the strands labeled $k$ and $k+1$ at $x_{m-1}$. Then

$$
d c_{m}=1+y_{k, k+1}^{m-1},
$$

and the matrix $\widetilde{Y}_{m-1}$ is most easily described entry by entry. Let

$$
\tau:\{1, \ldots, N(m)\} \rightarrow\{1, \ldots, N(m-1)\}, \quad \tau(i)= \begin{cases}i & \text { if } i<k \\ i+2 & \text { if } i \geq k .\end{cases}
$$

The $i, j$ entry of $\widetilde{Y}_{m-1}$ is given by $\tilde{y}_{i j}^{m-1}=y_{\tau(i), \tau(j)}^{m-1}+a_{i j}$, where

$$
a_{i j}=y_{i, k+1}^{m-1} y_{k, \tau(j)}^{m-1}+y_{i, k}^{m-1} c_{m} y_{k, \tau(j)}^{m-1}+y_{i, k+1}^{m-1} c_{m} y_{k+1, \tau(j)}^{m-1}+y_{i, k+1}^{m-1}\left(c_{m}\right)^{2} y_{k+1, \tau(j)}^{m-1}
$$

when $i<k \leq j$ and $a_{i j}=0$ otherwise.

Remark 5.2. The technique of adding some variation of splashes to simplify the differential first appeared in [Fuchs 2003] and has been used in several places in the literature. The version employed here is the same as that of [Fuchs and Rutherford 2011], to which we refer the reader for more details. For an alternate approach, we expect that a DGA of the same form would arise from iterating the "bordered Chekanov-Eliashberg algebra" construction introduced in [Sivek 2011].

5B. Proof of Theorem 1.2(2). We begin by introducing notation. Given an involution $\tau$ of $\{1, \ldots, N\}, \tau^{2}=i d$, we let $B_{\tau}=\left(b_{i j}\right)$ denote the $N \times N$ matrix with entries

$$
b_{i j}= \begin{cases}1 & \text { if } i<\tau(i)=j, \\ 0 & \text { else. }\end{cases}
$$

(B) $\Rightarrow$ (A). Suppose that $L$ the diagram admits a generalized normal ruling $\rho=$ $\left(\rho_{1}, \ldots, \rho_{m}\right)$. An augmentation $\varepsilon$ of the algebra $\mathscr{A}\left(L^{\prime}\right)$ is defined as follows: on all right cusps $c_{m}, \varepsilon\left(c_{m}\right)=0$; at crossings $b_{m}, \varepsilon\left(b_{m}\right)$ is 1 if $b_{m}$ is a switch and 0 otherwise; for all $m, \varepsilon\left(Y_{m}\right)=B_{\rho_{m}}$; and $\varepsilon\left(x_{i, j}^{m}\right)=0$ for all $i, j$ except when a switch occurs between $x_{m-1}$ and $x_{m}$. Assume the switch involves the $k$ and $k+1$ strands. If one of the switching strands is also a fixed point strand, then of the generators $x_{i j}^{m}$ augment only $x_{k, k+1}^{m}$. Else, note that due to the normality condition, near the switch the intervals connecting the switching strands and their companion strands (Remark 2.3) are either disjoint or nested. Assume that the switch occurs between the strands labeled $k$ and $k+1$. If the switch is disjoint, augment only $x_{k, k+1}^{m}$. If the switch is nested, augment $x_{k, k+1}^{m}$ and also $x_{\tau(k), \tau(k+1)}^{m}$ or $x_{\tau(k+1), \tau(k)}^{m}$, depending on whether $\tau(k)<\tau(k+1)$ or $\tau(k+1)<\tau(k)$. 
It is straightforward to verify from the formulas of the previous section that $\varepsilon$ is an augmentation. If $\rho$ is $p$-graded with respect to a Maslov potential $\mu$, then $\varepsilon$ is as well.

$(A) \Rightarrow(B)$. The proof of the reverse implication is based on some canonical form results from linear algebra due to Barannikov [1994].

Definition 5.3. An $M$-complex, $(V, \mathscr{B}, d)$ is a vector space $V$ over a field $\mathbb{F}$ with a chosen ordered basis $\mathscr{B}=\left\{v_{1}, \ldots, v_{N}\right\}$ together with a differential $d: V \rightarrow V$, $d^{2}=0$, of the form $d v_{i}=\sum_{i<j} c_{i j} v_{j}$.

Proposition 5.4. If $(V, \mathscr{B}, d)$ is an $M$-complex, then there exists a triangular change of basis $\left\{\tilde{v}_{1}, \ldots, \tilde{v}_{N}\right\}$, with $\tilde{v}_{i}=\sum_{i \leq j} a_{i j} v_{j}$, and an involution

$$
\tau:\{1, \ldots, N\} \rightarrow\{1, \ldots, N\}
$$

such that

$$
d \tilde{v}_{i}= \begin{cases}\tilde{v}_{j} & \text { if } i<\tau(i)=j \\ 0 & \text { else. }\end{cases}
$$

Moreover, the involution $\tau$ is unique.

Remark 5.5. (i) Suppose in addition that the basis elements $v_{i}$ are assigned degrees $\left|v_{i}\right| \in \mathbb{Z} / p$ so that $V$ is $\mathbb{Z} / p$-graded and $d$ has degree -1 . Then, the change of basis may be assumed to preserve degree. Hence, if $i<\tau(i)=j$, then $\left|v_{i}\right|=\left|v_{j}\right|+1$.

(ii) The classes $\left[\tilde{v}_{i}\right]$ such that $\tau(i)=i$ form a basis for the homology $H(V, d)$.

(iii) Proposition 5.4 has the following matrix interpretation: There is a unique function, $D \mapsto \tau(D)$ which assigns to every strictly upper triangular $N \times N$ matrix $D$ with $D^{2}=0$ an involution $\tau=\tau(D)$ such that there exists an invertible upper triangular matrix $P$ so that $P D P^{-1}=B_{\tau}$. Notice that the uniqueness assertion implies that $\tau\left(Q D Q^{-1}\right)=\tau(D)$ if $Q$ is nonsingular and upper triangular.

Proposition 5.6 [Barannikov 1994]. Suppose that $(V, \mathscr{B}, d)$ is an $M$-complex, and $k \in\{1, \ldots, N\}$ is such that $d v_{k}=\sum_{k+1<j} c_{k j} v_{j}$ so that the triple $\left(V, \mathscr{B}^{\prime}, d\right)$ with $\mathscr{B}^{\prime}=\left\{v_{1}, \ldots, v_{k+1}, v_{k}, \ldots, v_{N}\right\}$ is also an $M$-complex. Then, the associated involutions $\tau$ and $\tau^{\prime}$ are related as follows.

(1) It is always possible to have $\tau^{\prime}=(k k+1) \circ \tau \circ(k k+1)$, where $(k k+1)$ denotes the transposition.

(2) In the following cases, it is also possible to have $\tau^{\prime}=\tau$ :

(a) If $\tau(k+1)<\tau(k)<k<k+1$, or

$$
\begin{aligned}
& \tau(k)<k<k+1<\tau(k+1), \text { or } \\
& k<k+1<\tau(k+1)<\tau(k) .
\end{aligned}
$$

(b) If $\tau(k)<k<k+1=\tau(k+1)$ or $\tau(k)=k<k+1<\tau(k+1)$.

(c) If $\tau(k)=k<k+1=\tau(k+1)$. 
Remark 5.7. (i) From the matrix perspective, Proposition 5.6 puts restrictions on $\tau\left(P_{k, k+1} D P_{k, k+1}\right)$ when $P_{k, k+1}$ is the permutation matrix of the transposition $(k k+1)$ and the $k, k+1$-entry of $D$ is 0 .

(ii) Propositions 5.4 and 5.6 are essentially the same as Lemma 2 and Lemma 4 of [Barannikov 1994]. Proposition 5.6 is proven quite directly by considering cases.

Proof of $(\mathbf{A}) \Rightarrow(\mathbf{B})$. Suppose now that $\varepsilon$ is an augmentation of $\mathscr{A}\left(L^{\prime}\right)$.

For each $m$, the matrix $\varepsilon\left(Y_{m}\right)$ is strictly upper triangular and satisfies

$$
\left[\varepsilon\left(Y_{m}\right)\right]^{2}=\varepsilon \circ d\left(Y_{m}\right)=0 .
$$

Letting $\tau_{m}=\tau\left(\varepsilon\left(Y_{m}\right)\right)$ as in Remark 5.5 produces a sequence, $\tau_{1}, \ldots, \tau_{M}$, with $\tau_{m}$ an involution of $\{1, \ldots, N(m)\}$. We show that $\tau=\left(\tau_{1}, \ldots, \tau_{M}\right)$ satisfies the requirements of a generalized normal ruling. This requires establishing that the restrictions provided by Definitions 2.2 and 2.4 on consecutive involutions $\tau_{m-1}$ and $\tau_{m}$ are satisfied.

Recall that each interval $\left(x_{m-1}, x_{m}\right)$ contains a single crossing or cusp.

If $\left(x_{m-1}, x_{m}\right)$ contains a left cusp, then (5-2) and the definition of augmentation allow us to compute

$$
\varepsilon\left(Y_{m}\right)=\left(I+\varepsilon\left(X_{m}\right)\right) \varepsilon\left(\tilde{Y}_{m-1}\right)\left(I+\varepsilon\left(X_{m}\right)\right)^{-1} .
$$

Using Remark 5.5 we conclude that

$$
\tau_{m}=\tau\left(\varepsilon\left(Y_{m}\right)\right)=\tau\left(\varepsilon\left(\tilde{Y}_{m-1}\right)\right) .
$$

The $M$-complex associated with $\varepsilon\left(\tilde{Y}_{m-1}\right)$ is related to that of $\varepsilon\left(Y_{m-1}\right)$ by adding two new generators $v_{k}$ and $v_{k+1}$ to $\mathscr{B}$. The complex is the split extension of that of $\varepsilon\left(Y_{m-1}\right)$ by $\operatorname{span}\left\{v_{k}, v_{k+1}\right\}$ with the differential $d v_{k}=v_{k+1}$. It can then be checked from the definition that the involutions $\tau_{m-1}$ and $\tau_{m}$ satisfy Definition 2.2(2).

If $\left(x_{m-1}, x_{m}\right)$ contains a right cusp, let

$$
\mathscr{C}=\left(V_{m-1}, \mathscr{B}=\left\{v_{i} \mid i=1, \ldots, N(m-1)\right\}, d\right)
$$

denote the $M$-complex associated with the matrix $\varepsilon\left(Y_{m-1}\right)$ by the formula

$$
d v_{i}=\sum_{i<j} \varepsilon\left(y_{i j}^{m-1}\right) v_{j} .
$$

Note that $\tau_{m-1}$ is precisely the involution associated to $\mathscr{C}$ by Proposition 5.4. From $0=\varepsilon \circ d\left(c_{m}\right)$ we deduce that $1=\varepsilon\left(y_{k, k+1}^{m-1}\right)$, and it follows that $\tau_{m-1}(k)=k+1$.

Next, one observes that $\varepsilon\left(\widetilde{Y}_{m-1}\right)$ is the matrix of the $M$-complex

$$
\widetilde{\mathscr{C}}=\left(\widetilde{V}_{m-1}, \widetilde{\mathscr{B}}=\left\{\left[v_{i}\right] \mid i \neq k, k+1\right\}, \tilde{d}\right),
$$


where $\widetilde{V}_{m-1}$ is the quotient of $V_{m-1}$ by the subcomplex

$$
\left\{v_{k}+\varepsilon\left(c_{m}\right) v_{k+1}, d\left(v_{k}+\varepsilon\left(c_{m}\right) v_{k+1}\right)\right\}
$$

and $\tilde{d}$ is the differential induced by $d$. If $\left\{\tilde{v}_{i}\right\}$ is a triangular change of basis for $\mathscr{C}$ satisfying the conditions of Proposition 5.4, then $\left\{\left[\tilde{v}_{i}\right] \mid i \neq k, k+1\right\}$ will be such a basis for $\widetilde{\mathscr{C}}$, so that the involution associated with $\varepsilon\left(\widetilde{Y}_{m-1}\right)$ is related to $\tau_{m-1}$ as required in Definition 2.2(3). Finally, we get $\tau_{m}=\tau\left(\varepsilon\left(Y_{m}\right)\right)=\tau\left(\varepsilon\left(\tilde{Y}_{m-1}\right)\right)$ by using (5-3).

If $\left(x_{m-1}, x_{m}\right)$ contains a crossing $b_{m}$, we have $0=\varepsilon \circ d\left(b_{m}\right)=\varepsilon\left(y_{k, k+1}^{m-1}\right)$. Thus, $\varepsilon\left(\widehat{Y}_{m-1}\right)=\varepsilon\left(Y_{m-1}\right)$ with both matrices having 0 as their $(k, k+1)$ entry. Then, compute that

$$
\begin{aligned}
\varepsilon\left(B_{k, k+1}\right) \varepsilon\left(\widehat{Y}_{m-1}\right) \varepsilon & \left(B_{k, k+1}^{-1}\right) \\
& =P_{k, k+1}\left[I+\varepsilon\left(b_{m}\right) E_{k, k+1}\right] \varepsilon\left(Y_{m-1}\right)\left[I+\varepsilon\left(b_{m}\right) E_{k, k+1}\right] P_{k, k+1} .
\end{aligned}
$$

Regardless of the value of $\varepsilon\left(b_{m}\right)$, the $(k, k+1)$-entry of

$$
\left[I+\varepsilon\left(b_{m}\right) E_{k, k+1}\right] \varepsilon\left(Y_{m-1}\right)\left[I+\varepsilon\left(b_{m}\right) E_{k, k+1}\right]
$$

is 0 , so the matrix $A=\varepsilon\left(B_{k, k+1}\right) \varepsilon\left(\widehat{Y}_{m-1}\right) \varepsilon\left(B_{k, k+1}^{-1}\right)$ is strictly upper triangular and $\tau(A)$ is related to

$$
\tau\left(\left(I+\varepsilon\left(b_{m}\right) E_{k, k+1}\right) \varepsilon\left(Y_{m-1}\right)\left(I+\varepsilon\left(b_{m}\right) E_{k, k+1}\right)\right)=\tau\left(Y_{m-1}\right)=\tau_{m-1}
$$

as in Proposition 5.6. It follows that

$$
\tau_{m}=\tau\left(\varepsilon\left(Y_{m}\right)\right)=\tau\left(\left(I+\varepsilon\left(X_{m}\right)\right) A\left(I+\varepsilon\left(X_{m}\right)\right)^{-1}\right)=\tau(A)
$$

and $\tau_{m-1}$ satisfy the requirements near crossings (including the normality conditions) of Definition 2.4.

The statement that $\tau$ is $p$-graded if $\varepsilon$ is $p$-graded follows from (i) of Remark 5.5. As in (5-4), $\varepsilon\left(Y_{m}\right)$ is the matrix of an $M$-complex with basis $v_{1}, \ldots, v_{N(m)}$ corresponding to the strands of $L$ at $x_{m}$. If $\varepsilon$ is $p$-graded with respect to $\mu$, then we can assign a grading by $\left|v_{i}\right|=\mu(m, i)$ and the differential will have degree -1 .

\section{Acknowledgements}

This work was initiated through the PRUV program at Duke University. We thank David Kraines for supervising the program and encouraging our participation. Also, we thank Lenny $\mathrm{Ng}$ for his interest in the project. A portion of the writing was carried out while the second author was a visitor at the Max Planck Institute for Mathematics in Bonn, and it is a pleasure to acknowledge the MPIM for their hospitality. 


\section{References}

[Barannikov 1994] S. A. Barannikov, "The framed Morse complex and its invariants", pp. 93-115 in Singularities and bifurcations, edited by V. I. Arnold, Adv. Soviet Math. 21, Amer. Math. Soc., Providence, RI, 1994. MR 96a:57065 Zbl 0996.57514

[Chekanov 2002] Y. Chekanov, "Differential algebra of Legendrian links", Invent. Math. 150:3 (2002), 441-483. MR 2003m:53153 Zbl 1029.57011

[Chekanov and Pushkar 2005] Y. V. Chekanov and P. E. Pushkar, "Combinatorics of fronts of Legendrian links, and Arnold's 4-conjectures”, Uspekhi Mat. Nauk 60:1(361) (2005), 99-154. In Russian; translated in Russian Math. Surveys 60:1 (2005), 95-149. MR 2006h:58049 Zbl 1085.57008

[Chmutov and Goryunov 1997] S. Chmutov and V. Goryunov, "Polynomial invariants of Legendrian links and plane fronts", pp. 25-43 in Topics in singularity theory, edited by A. Khovanskiı̌ et al., Amer. Math. Soc. Transl. Ser. 2 180, Amer. Math. Soc., Providence, RI, 1997. MR 2002c:57015 Zbl 0886.57005

[Cornwell 2012a] C. Cornwell, "Bennequin type inequalities in lens spaces", Int. Math. Res. Not. 2012:8 (2012), 1890-1916. Zbl 06034372 arXiv 1002.1546

[Cornwell 2012b] C. Cornwell, "A polynomial invariant for links in lens spaces", J. Knot Theory Ramifications 21:6 (2012), \# 1250060. Zbl 06025162 arXiv 1002.1543v2

[Ding and Geiges 2010] F. Ding and H. Geiges, "Legendrian helix and cable links", Commun. Contemp. Math. 12:3 (2010), 487-500. MR 2011j:53174 Zbl 1202.53082

[Fuchs 2003] D. Fuchs, "Chekanov-Eliashberg invariant of Legendrian knots: existence of augmentations”, J. Geom. Phys. 47:1 (2003), 43-65. MR 2004h:57007 Zbl 1028.57005

[Fuchs and Ishkhanov 2004] D. Fuchs and T. Ishkhanov, "Invariants of Legendrian knots and decompositions of front diagrams”, Mosc. Math. J. 4 (2004), 707-717. MR 2005i:57033 Zbl 1073.53106

[Fuchs and Rutherford 2011] D. Fuchs and D. Rutherford, "Generating families and Legendrian contact homology in the standard contact space", J. Topol. 4:1 (2011), 190-226. MR 2012e:57046 Zbl 1237.57026

[Fuchs and Tabachnikov 1997] D. Fuchs and S. Tabachnikov, "Invariants of Legendrian and transverse knots in the standard contact space", Topology 36:5 (1997), 1025-1053. MR 99a:57006 Zbl 0904.57006

[Geiges 2008] H. Geiges, An introduction to contact topology, Cambridge Studies in Advanced Mathematics 109, Cambridge University Press, 2008. MR 2008m:57064 Zbl 1153.53002

[Licata 2011] J. E. Licata, "Invariants for Legendrian knots in lens spaces", Commun. Contemp. Math. 13:1 (2011), 91-121. MR 2772580 Zbl 05864098

[Ng and Traynor 2004] L. Ng and L. Traynor, "Legendrian solid-torus links", J. Symplectic Geom. 2:3 (2004), 411-443. MR 2005k:57051 Zbl 1097.57014

[Rutherford 2006] D. Rutherford, "Thurston-Bennequin number, Kauffman polynomial, and ruling invariants of a Legendrian link: the Fuchs conjecture and beyond", Int. Math. Res. Not. 2006 (2006), Art. ID 78591. MR 2007a:57020 Zbl 1106.57012

[Rutherford 2011] D. Rutherford, "HOMFLY-PT polynomial and normal rulings of Legendrian solid torus links", Quantum Topol. 2:2 (2011), 183-215. MR 2783131 Zbl 1230.57016

[Sabloff 2005] J. M. Sabloff, "Augmentations and rulings of Legendrian knots", Int. Math. Res. Not. 2005:19 (2005), 1157-1180. MR 2006e:57010 Zbl 1082.57020

[Sivek 2011] S. Sivek, "A bordered Chekanov-Eliashberg algebra", J. Topol. 4:1 (2011), 73-104. MR 2783378 Zbl 1219.57022 
[Traynor 1997] L. Traynor, "Legendrian circular helix links", Math. Proc. Cambridge Philos. Soc. 122:2 (1997), 301-314. MR 98f:58085 Zbl 0902.57006

[Turaev 1988] V. G. Turaev, "The Conway and Kauffman modules of a solid torus", Zap. Nauchn. Sem. Leningrad. Otdel. Mat. Inst. Steklov. (LOMI) 167:Issled. Topol. 6 (1988), 79-89. In Russian; translated in J. Soviet Math. 52 (1990), 2799-2805. MR 90f:57012 Zbl 0673.57004

Received October 4, 2011. Revised December 8, 2011.

MiKHAIL LAVROV

CARnegie Mellon University

PITTSBURGH, PA 15213

UNITED STATES

mlavrov@andrew.cmu.edu

DAN RUTHERFORD

UNIVERSITY OF ARKANSAS

FAYETTEVILLE, AR 72701

UNITED STATES

drruther@uark.edu 


\title{
PACIFIC JOURNAL OF MATHEMATICS
}

\author{
http://pacificmath.org \\ Founded in 1951 by \\ E. F. Beckenbach (1906-1982) and F. Wolf (1904-1989)
}

\section{EDITORS}

V. S. Varadarajan (Managing Editor)

Department of Mathematics

University of California

Los Angeles, CA 90095-1555

pacific@math.ucla.edu

Vyjayanthi Chari

Department of Mathematics

University of California

Riverside, CA 92521-0135

chari@math.ucr.edu

\section{Robert Finn}

Department of Mathematics Stanford University

Stanford, CA 94305-2125

finn@math.stanford.edu

Kefeng Liu

Department of Mathematics

University of California

Los Angeles, CA 90095-1555

liu@math.ucla.edu
Darren Long

Department of Mathematics

University of California

Santa Barbara, CA 93106-3080

long@math.ucsb.edu

Jiang-Hua Lu

Department of Mathematics

The University of Hong Kong

Pokfulam Rd., Hong Kong jhlu@maths.hku.hk

Alexander Merkurjev

Department of Mathematics

University of California

Los Angeles, CA 90095-1555

merkurev@math.ucla.edu
Sorin Popa

Department of Mathematics University of California

Los Angeles, CA 90095-1555 popa@math.ucla.edu

Jie Qing

Department of Mathematics

University of California

Santa Cruz, CA 95064

qing@cats.ucsc.edu

Jonathan Rogawski

Department of Mathematics

University of California

Los Angeles, CA 90095-1555

jonr@math.ucla.edu

\section{PRODUCTION}

pacific@math.berkeley.edu

\section{SUPPORTING INSTITUTIONS}

ACADEMIA SINICA, TAIPEI

CALIFORNIA INST. OF TECHNOLOGY INST. DE MATEMÁTICA PURA E APLICADA KEIO UNIVERSITY

MATH. SCIENCES RESEARCH INSTITUTE NEW MEXICO STATE UNIV.

OREGON STATE UNIV.

\author{
STANFORD UNIVERSITY \\ UNIV. OF BRITISH COLUMBIA \\ UNIV. OF CALIFORNIA, BERKELEY \\ UNIV. OF CALIFORNIA, DAVIS \\ UNIV. OF CALIFORNIA, LOS ANGELES \\ UNIV. OF CALIFORNIA, RIVERSIDE \\ UNIV. OF CALIFORNIA, SAN DIEGO \\ UNIV. OF CALIF., SANTA BARBARA
}

\author{
UNIV. OF CALIF., SANTA CRUZ \\ UNIV. OF MONTANA \\ UNIV. OF OREGON \\ UNIV. OF SOUTHERN CALIFORNIA \\ UNIV. OF UTAH \\ UNIV. OF WASHINGTON \\ WASHINGTON STATE UNIVERSITY
}

These supporting institutions contribute to the cost of publication of this Journal, but they are not owners or publishers and have no responsibility for its contents or policies.

See inside back cover or pacificmath.org for submission instructions.

The subscription price for 2012 is US \$420/year for the electronic version, and \$485/year for print and electronic.

Subscriptions, requests for back issues from the last three years and changes of subscribers address should be sent to Pacific Journal of Mathematics, P.O. Box 4163, Berkeley, CA 94704-0163, U.S.A. Prior back issues are obtainable from Periodicals Service Company, 11 Main Street, Germantown, NY 12526-5635. The Pacific Journal of Mathematics is indexed by Mathematical Reviews, Zentralblatt MATH, PASCAL CNRS Index, Referativnyi Zhurnal, Current Mathematical Publications and the Science Citation Index.

The Pacific Journal of Mathematics (ISSN 0030-8730) at the University of California, c/o Department of Mathematics, 969 Evans Hall, Berkeley, CA 94720-3840, is published monthly except July and August. Periodical rate postage paid at Berkeley, CA 94704, and additional mailing offices. POSTMASTER: send address changes to Pacific Journal of Mathematics, P.O. Box 4163, Berkeley, CA 94704-0163.

PJM peer review and production are managed by EditFLOW ${ }^{\mathrm{TM}}$ from Mathematical Sciences Publishers.

PUBLISHED BY PACIFIC JOURNAL OF MATHEMATICS

at the University of California, Berkeley 94720-3840

A NON-PROFIT CORPORATION

Typeset in LATEX

Copyright $(02012$ by Pacific Journal of Mathematics 


\section{PACIFIC JOURNAL OF MATHEMATICS}

Volume $258 \quad$ No. $2 \quad$ August 2012

Uniqueness theorems for $\mathrm{CR}$ and conformal mappings

YounG-JUn CHOI and JAE-CHEON JOO

Some finite properties for vertex operator superalgebras

269

CHONGYING DONG and JIANZHI HAN

On the geometric flows solving Kählerian inverse $\sigma_{k}$ equations

291

HAO FANG and MIJIA LAI

An optimal anisotropic Poincaré inequality for convex domains

GUOFANG WANG and CHAO XIA

Einstein metrics and exotic smooth structures

MASASHI ISHIDA

Noether's problem for $\hat{S}_{4}$ and $\hat{S}_{5}$

MING-CHANG KANG and JiAn ZHOU

Remarks on the behavior of nonparametric capillary surfaces at corners 369

KIRK E. LANCASTER

Generalized normal rulings and invariants of Legendrian solid torus links

MIKHAIL LAVROV and DAN RUTHERFORD

Classification of singular $\mathbb{Q}$-homology planes II: $\mathbb{C}^{1}$ - and $\mathbb{C}^{*}$-rulings.

KAROL PALKA

A dynamical interpretation of the profile curve of CMC twizzler surfaces

OSCAR M. PERDOMO

Classification of Ising vectors in the vertex operator algebra $V_{L}^{+}$

HIROKI SHIMAKURA

Highest-weight vectors for the adjoint action of $\mathrm{GL}_{n}$ on polynomials 